\title{
Effects of in vivo cyclic compressive loading on the distribution of local Col2 and superficial lubricin in rat knee cartilage
}

\author{
AUTHOR(S): \\ Ji, Xiang; Ito, Akira; Nakahata, Akihiro; Nishitani, \\ Kohei; Kuroki, Hiroshi; Aoyama, Tomoki
}

\section{CITATION:}

$\mathrm{Ji}$, Xiang ... [et al]. Effects of in vivo cyclic compressive loading on the distribution of local Col2 and superficial lubricin in rat knee cartilage. Journal of Orthopaedic Research 2021, 39(3): 543-552

\section{ISSUE DATE:}

2021-03

URL:

http://hdl.handle.net/2433/263335

\section{RIGHT:}

This is the peer reviewed version of the following article: [Journal of Orthopaedic Research, 39(3), 543-552, March 2021], which has been published in final form at https://doi.org/10.1002/jor.24812. This article may be used for non-

commercial purposes in accordance with Wiley Terms and Conditions for Self-Archiving.; The full-text file will be made open to the public on 04 August 2021 in accordance with publisher's 'Terms and Conditions for Self-Archiving'.; This is not the published version. Please cite only the published version. この論文は出版蝂でありません。引用の際には出 版社版をご確認ご利用ください。 
1 Effects of in vivo cyclic compressive loading on the distribution of local Col2 and superficial

2 lubricin in rat knee cartilage

3

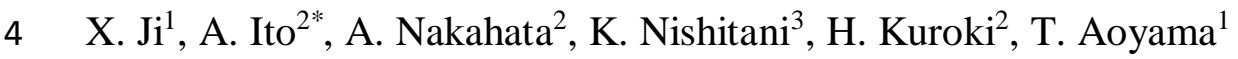

5

$6{ }^{1}$ Department of Development and Rehabilitation of Motor Function, Human Health Sciences, Graduate

$7 \quad$ School of Medicine, Kyoto University

$8 \quad{ }^{2}$ Department of Motor Function Analysis, Human Health Sciences, Graduate School of Medicine, Kyoto

9 University

$10{ }^{3}$ Department of Orthopaedic Surgery, Graduate School of Medicine, Kyoto University

11

12 Author E-mail addresses

13 gochickenjp@gmail.com (XJ), ito.akira.4m@kyoto-u.ac.jp (AI), akihironakahata@gmail.com (AN),

14 nkohei@kuhp.kyoto-u.ac.jp (KN), kuroki.hiroshi.6s@kyoto-u.ac.jp (HK), aoyama.tomoki.4e@kyoto-

$15 \quad$ u.ac.jp (TA)

16

17 *Corresponding author:

$18 \quad$ Akira Ito

1953 Shogoin, Kawahara-cho, Sakyo-ku, Kyoto 606-8507, Japan

20 Tel: +81-75-751-3964; Fax: +81-75-751-3964

21 E-mail: ito.akira.4m@kyoto-u.ac.jp

22 Running headline: Cyclic loading affects Col2, lubricin 


\section{Abstract}

24 This study aimed to examine the effects of an episode of in vivo cyclic loading on rat knee articular 25 cartilage (AC) under medium-term observation, while also investigating relevant factors associated with 26 the progression of post-traumatic osteoarthritis (PTOA). Twelve-week-old Wistar rats underwent one 27 episode comprising 60 cycles of $20 \mathrm{~N}$ or $50 \mathrm{~N}$ dynamic compression on the right knee joint.

28 Spatiotemporal changes in the AC after loading were evaluated using histology and

29 immunohistochemistry at 3 days and 1, 2, 4, and 8 weeks after loading ( $\mathrm{n}=6$ for each condition).

30 Chondrocyte vitality was assessed at $1,3,6$, and $12 \mathrm{~h}$ after loading ( $\mathrm{n}=2$ for each condition). A localized

31 AC lesion on the lateral femoral condyle was confirmed in all subjects. The surface and intermediate

32 cartilage in the affected area degenerated after loading, but the calcified cartilage remained intact.

33 Expression of type II collagen in the lesion cartilage was upregulated after loading, whereas the

34 superficial lubricin layer was eroded in response to cyclic compression. However, the distribution of 35 superficial lubricin gradually recovered to the normal level 4 weeks after loading-induced injury. We confirmed that 60 repetitions of cyclic loading exceeding $20 \mathrm{~N}$ could result in cartilage damage in the rat

37 knee. Endogenous repairs in well-structured joints work well to rebuild protective layers on the lesion cartilage surface, which may be the latent factor delaying the progression of PTOA.

43 Keywords: In vivo cyclic compression; Post-traumatic osteoarthritis; Cartilage degeneration; Rat model; 44 Type II collagen; Superficial lubricin 


\section{Introduction}

46 Post-traumatic osteoarthritis (PTOA) is a classification of clinical osteoarthritis (OA) that is common

47 among patients with a history of articular cartilage (AC) damage and ligament injury. Animal models play

48 an important role in understanding the pathophysiology of PTOA as well as developing novel therapies to

49 treat this disease. Small animals such as rodents have the advantage of a faster pathological process and

50 lower maintenance costs compared to large animals; hence, rodents are widely used as PTOA models for

51 experimental purposes. The anterior cruciate ligament transection (ACLT) and destabilization of the

52 medial meniscus (DMM) were the optimal conditions for short-term studies in the past. In recent decades,

53 non-surgical models have been considered instead in order to avoid surgery-induced inflammation that

54 could affect the evaluation. One of the most promising candidates is cyclic compression on knee $\mathrm{AC}^{1}$.

56 The in vivo cyclic compression model was first designed for verifying trabecular bone adaptation to

57 mechanical loading ${ }^{2-4}$ and was developed as a nonsurgical model of OA in later studies ${ }^{5-12}$. However, there

58 are doubts regarding the accuracy of the simulated pathologic progression of clinically relevant secondary

59 OA in these models. One problem was over-frequent loading, which contributed to excessive subchondral

60 bone reaction and the formation of disproportionately giant osteophytes, reported in many cases ${ }^{7,8,12}$. Ko

61 et $\mathrm{al}^{11}$ reported a single session of loading induced OA-like morphological destruction, where the regimen

62 comprised 1200 cycles, roughly equal to the 5 days/week design in other studies. Poulet et al ${ }^{5}$ confirmed

64 for 2 weeks found no loss in Safranin O staining. Therefore, further study on long-term tracking of low65 dose loading effectiveness is necessary. 
67 Although earlier studies using cyclic compression models showed visible osteophytes and reduced

68

69

70

71

72

73

74

75

76

77

78

79

80

81

82

83

84

85

86

87

88

substrate staining, none reported irregular wearing of cartilage surface or subchondral bone porosity loss, which are important characteristics of OA progression in surgery-induced rodent OA models ${ }^{13,14}$. Thus, depending on the magnitude of compressive loading and the methodology of joint instability surgery, the mechanisms of repair or alleviation of the AC lesion in OA progression are still unclear.

The current study aimed to track the relatively long-term effect of in vivo low-dose cyclic loading on rat knee joints, the first such study in rat species. Further, we examined changes over time in loading-affected cartilage and investigated potential causes for the slower progression of OA development in a non-surgical model relative to a surgical model.

\section{Methods}

\section{Mechanical loading procedures and sample allocation}

All experimental procedures were approved by the animal research committee of Kyoto University (approval number: Med kyo 17616). Seventy-four 12-week-old wild-type male Wistar rats were used in this study. The animals were anesthetized with 5\% isoflurane solution (Pfizer, Tokyo, Japan) before being injected intraperitoneally with $1 \mu \mathrm{g} / \mathrm{g}$ somnopentyl (Kyoritsu Seiyaku Corp., Tokyo, Japan). Each animal's right knee was fixed with a customized cup with approximately 140 degrees flexion, as previously described $^{6}$, and subjected to one session of dynamic loading in the daytime using a measuring compression instrument (Autograph AG-X, Shimazu, Japan). The loading regimen included a preload of $5 \mathrm{~N}$ and peak load of $20 \mathrm{~N}$ or $50 \mathrm{~N}$, with approaching speed of $1 \mathrm{~mm} / \mathrm{s}$ and $10 \mathrm{~s}$ rest intervals (Figure 1A-B). The load levels were set according to previous studies in other species ${ }^{6,9}$, and were proportionately amplified based 
89

90

91

92

93

94

95

96

97

98

99

100

101 102

103

104

105

106

107

108

109

110

on animal weight. Each session comprised 60 cycles that lasted approximately 12 min total. After loading compression, animals were returned to transparent plastic cages with a 12-h light/dark cycle and provided adequate feed and free space for movement. Experimental rats were randomly divided into three groups (peak load $20 \mathrm{~N}$ or $50 \mathrm{~N}$, and control). The rats ( $\mathrm{n}=6$ for each condition, $\mathrm{n}=60$ in total) were sacrificed for histological analysis at 3 days and 1, 2, 4, and 8 weeks after compression. Knee samples that underwent $20 \mathrm{~N}$ loading were harvested at 1, 3, 6, and $12 \mathrm{~h}$ for the live/dead assessment of chondrocytes ( $\mathrm{n}=2 \mathrm{for}$ each timepoint, $\mathrm{n}=8$ in total). Normal 12 -week old Wistar rat samples served as controls $(\mathrm{n}=4)$ for histological analysis and controls $(n=2)$ for cell viability evaluation (Figure 1C). Randomization was performed using Excel functions, and animals in different experimental groups were treated in random order each time.

\section{Live/dead analysis of chondrocytes}

To evaluate the live/dead spatiotemporal changes of chondrocytes on lateral femoral condyles, calcein AM/ethd-1 staining (LIVE/DEAD Viability/Cytotoxicity Kit, Thermo Fisher Scientific, Tokyo, Japan) was performed immediately after specimens were dissected from the knee joints. Samples were treated with calcein AM (diluted 1:500) and Ethd-1 (diluted 1:4000) solutions in PBS for 20 min at room temperature. Samples were then rinsed in PBS and cut into two parts of the femoral intercondylar sulcus. The lateral half was then mounted on a transparent plate with the femoral condyle towards the camera (Supplementary figure 1). Fluorescence micrographs were taken using a fluorescence microscope (Fluoview FV10i, Olympus, Tokyo, Japan) with FITC (495/519 nm) and PI (535/617 nm) channels. Live cells were indicated by green fluorescence, and dead cells by red fluorescence. Contralateral limbs harvested at $12 \mathrm{~h}$ were used as controls. 
113 Knee joints were fixed in $4 \%$ paraformaldehyde overnight and decalcified in 10\% EDTA for 25 days.

114 Samples were then embedded in paraffin. Twelve $6-\mu \mathrm{m}$ sagittal sections for every $100-\mu \mathrm{m}$ interval were 115 prepared, covering the entire area of the lesion in the lateral femur for each sample. Safranin O, fast green, 116 and hematoxylin staining were performed on each section, and the average modified Mankin score ${ }^{15}$ was 117 calculated to evaluate the degree of cartilage degeneration at the lateral femoral condyle. To assess the 118 volume of degenerative cartilage, the lesion area was defined using ImageJ software, and the stacked 119 volume was calculated by multiplying the total area by a $100-\mu \mathrm{m}$ average thickness. The intensity of 120 Safranin O staining was calculated on an inverted 8-bit grayscale image using ImageJ software. The 121 relative intensity in lesion areas was calculated by dividing by the intensity in normal cartilage 122 (Supplementary figure 2). In addition, the hematoxylin-stained nuclei of chondrocytes in the lesion 123 cartilage were counted.

126 Immunohistochemical staining of type II collagen (Fine Chemical Co., Toyama, Japan; diluted 1:200), 127 matrix metalloproteinase thirteen (MMP-13) (ab39012 Abcam Co., Tokyo, Japan; diluted 1:1000), A 128 disintegrin and metalloproteinase with thrombospondin motifs five (ADAMTS-5) (ab185795 Abcam Co., 129 Tokyo, Japan; diluted 1:50), and Lubricin/Proteoglycan 4 (EMD Millipore, Temecula, USA; diluted 130 1:1000) were performed as described below. Deparaffinized sections were treated with $3 \%$ hydrogen 131 peroxide solution for $30 \mathrm{~min}$. Sections were then stained for the anti-type II collagen reaction and treated 132 with $1.25 \%$ hyaluronidase for $60 \mathrm{~min}$ at room temperature. Sections for the ADAMTS-5 reaction were 
133 treated with HistoVT One solution (Nacalai Tesque, Inc., Kyoto, Japan; diluted 1:10) for 40 min at $65^{\circ} \mathrm{C}$.

134 After rinsing in PBS, nonspecific reactions were suppressed by blocking with 5\% normal goat serum for

13560 min. Subsequently, sections were treated with primary antibodies and incubated at $4{ }^{\circ} \mathrm{C}$ overnight.

136 Sections were then washed in PBS and treated with goat anti-rabbit IgG (MMP-13 and ADAMTS-5) or

137 goat anti-mouse IgG (type II collagen and lubricin) for $30 \mathrm{~min}$ at room temperature. Detection was

138 performed using the streptavidin-biotin-peroxidase complex technique with an Elite ABC kit (diluted

139 1:100; Vector Laboratories, Burlingame, CA, USA). Localization was detected using 3,3-

140 diaminobenzidine solution (Vector Laboratories) followed by counterstaining with hematoxylin.

142 Immunohistological staining in the cartilage matrix of type II collagen and lubricin was evaluated using

143 ImageJ software. Images were converted to grayscale (0-255) from dark to bright, and intensity was 144 calculated by subtracting the values in blank spaces. Details of ROI selection are described in 145 Supplementary figure 3. The number of MMP-13- and ADAMTS-5-positive immunostained 146 chondrocytes in the lesion area and the adjoining zone were counted and normalized by dividing by the 147 corresponding cartilage surface length. The adjoining zone was defined as the area in proximity to the 148 lesion cartilage in a $0.48 \mathrm{~mm} \times 0.64 \mathrm{~mm} 200 \times$ histological image.

Statistical analysis

151 Statistical analyses were performed using SPSS software (version 22.0; SPSS Inc., Chicago IL). Two-way 152 analysis of variance was employed to analyze histological staining with loading as intragroup factors and 153 duration as intergroup factors. The normality of all continuous data was examined using the Shapiro-Wilk 154 normality test. The parametric variables of the modified Mankin score, volume of degenerative areas, and 
155 semi-quantitative measurements of immunohistochemistry were included in the model directly, whereas 156 the nonparametric variables were first transformed into ranked data and then introduced into the model. 157 Comparisons between intergroup marginal means using Tukey HSD tests were performed only when the 158 main effects exhibited significant results. When the analysis showed interactional effects in addition to 159 the significant main effects, multiple one-way ANOVA tests with post-hoc comparisons for stratified 160 samples were conducted on each level to examine potential differences in interactional effects among the 161 levels. Additionally, Mann-Whitney U (2 groups) or Kruskal-Wallis H tests (3 groups) were applied to 162 compare the control and loaded samples. The required sample size was calculated based on our pilot 163 experimental data of lesion area size between groups. P-value $<0.05$ was considered statistically 164 significant.

\section{Results}

\section{Vitality of chondrocytes after cyclic loading}

168 Samples that underwent $20 \mathrm{~N}$ cyclic compression were stained with calcein AM/ethd-1 and evaluated 169 (Supplementary figure 4). Representative images exhibited mixed distribution of red and green fluorescent 170 cells at 1 and $3 \mathrm{~h}$ after loading, whereas large areas without green-stained chondrocytes were observed at 1716 and $12 \mathrm{~h}$, indicating that complete cell death occurred within $6 \mathrm{~h}$, even at the lower load level of $20 \mathrm{~N}$.

174 Histology showed that AC in both groups was damaged, and one focal degenerative zone in the lateral 175 femoral condyle was confirmed for every subject (Supplementary figure 4A). However, the AC surface 
176 remained intact except for a slight fibrillation present in several samples (data not shown). A clear 177 boundary between the lesion cartilage and unaffected calcified cartilage was observed 2 weeks after 178 loading. The average modified Mankin score per section increased after loading (Supplementary figure $1794 \mathrm{~F}$ ) and differed between groups and observational durations (Supplementary figure 4B). As the peak load 180 or interval time increased, the degree of degeneration tended to worsen at higher histological scores 181 (Supplementary figure 4B). Although the lesion area volume did not change significantly throughout the 182 duration of the study, it was significantly higher in the $50 \mathrm{~N}$ load group than in the $20 \mathrm{~N}$ group at all time 183 points (Supplementary figure 4C). The relative Safranin O staining intensity in the lesion area declined 184 with time after loading for both groups compared to the intact area (Supplementary figure 4D); however, 185 there was no evident difference between groups with $20 \mathrm{~N}$ or $50 \mathrm{~N}$ peak loads. In addition, the number of 186 hematoxylin-stained nuclei in the lesion area continuously decreased after loading in both groups 187 (Supplementary figure 4E, 4G), whereas no significant changes were found in medium-term observation 188 from 2 to 8 weeks.

191 Immunohistochemistry results showed focal type II collagen overexpression in the AC lesion. Enhanced 192 staining was observed in each sample compared to adjacent intact substrates (Figure 3A), and the intensity 193 in loaded samples was significantly higher than in the control group (Figure 3B). However, there were no 194 notable effects on intensity in the lesion region with different load levels or time points (Figure 3C-D), 195 regardless of whether raw values or relative percentage increments were used. 
198 Superficial and intermediate zone chondrocytes in the control group moderately expressed MMP-13 and 199 ADAMTS-5 (Supplementary figure 5). In the loading groups, we found positively stained radial zone 200 chondrocytes under the lesion area (Supplementary figure 6, Figure 4) that were not observed in normal 201 samples (quantitative data not shown). The number of active cells in the area adjoining the lesion (no 202 direct contact) significantly increased after loading compared to normal AC (Supplementary figure 6B, 203 Figure 4B). Moreover, the results of semi-quantitative analysis revealed that the number of both MMP204 13- and ADAMTS-5-positive chondrocytes (in the lesion area or in the adjoining region) gradually 205 decreased during the 8-week observation period (Supplementary figure 6C-D, Figure 4C-D). However, 206 no significant main effects were generated by the load levels.

209 Lubricin expression in the lateral femoral condyle AC is presented in Figure 5. The staining intensity in 210 the lesion area of substrates within the superficial cartilage declined one week after loading compared to 211 that in the intact area (Figure 5A). However, semi-quantitative analyses showed an increased 212 concentration of lubricin on the AC lesion over time, reaching the same level at 4 and 8 weeks compared 213 to the intact area (Figure 5B). No statistical load-level effect was found, whereas the main effects of 214 duration and interaction between observational duration and load effect were confirmed. Furthermore, 215 results of stratified analysis revealed that superficial lubricin in $20 \mathrm{~N}$-loaded samples were more likely to 216 recover (more pairwise differences) in comparison to $50 \mathrm{~N}$ samples (Figure 5C-D). Additionally, we found 217 signs of stain aggregating around the site of degenerative chondrocytes or even lacunae from dead cells 218 lacking hematoxylin-stained nuclei. 


\section{Discussion}

221 The current study demonstrated for the first time that a single episode comprising 60 cycles of mechanical 222 stimulus can induce AC lesions in the lateral femoral condyle of rats, consistent with results reported in 223 smaller rodents such as mice ${ }^{5-6}$. Compared to surgical PTOA models of ACLT and DMM that gradually 224 develop chondrocyte apoptosis and cartilage matrix loss ${ }^{16}$, we did not find any apoptotic cells around the 225 lesion area, even at $6 \mathrm{~h}$ after loading (data not shown). This was considerably different from previous results in mice showing that clustered active TUNEL-stained chondrocytes were retained in degenerative 227 AC until 14 days after loading ${ }^{6}$. Our results from live/dead staining demonstrate that chondrocytes in the 228 superficial lesion cartilage were dead within $6 \mathrm{~h}$ due to direct damage (Figure 2). The Mankin score ${ }^{15}$ 229 deteriorated over time, whereas structural destruction of AC cartilage did not progress as rapidly as the 230 invasive models that showed a jagged cartilage surface and subchondral bone perforation within 4 weeks 231 after the instability surgery ${ }^{17,18}$. Furthermore, there was a distinct difference in staining between AC above 232 and below the tidemark (Supplementary figure 4A), consistent with in vitro results indicating that calcified 233 radial zones of cartilage suffered less than $5 \%$ of the total mechanical stress ${ }^{19,20}$. Cell signaling studies 234 have suggested that overloading activates the toll-like receptors expressed on chondrocytes, resulting in 235 the release of inflammatory cytokines ${ }^{21}$ and catalyzing ADAMTS-5 for aggrecan degradation ${ }^{22}$, consistent 236 with findings of the current study (Supplementary figure 6). According to our investigation, this non237 surgical model may be superior for simulation of acute extensive AC damage, which is common in athletic 238 injuries.

240 Expression of $\mathrm{Col} 2$ was found to be transiently increased within $1 \mathrm{~h}$ after ex vivo mechanical loading in 241 several experiments using extracted cartilage explants ${ }^{23,24}$. A recently published tissue-engineering 242 review ${ }^{25}$ summarized the biochemical anabolism of synthetic substrate-seeded chondrocytes subjected to 
243 in vitro dynamic loading, most of which demonstrated subsequent Col2 upregulation in response to 244 various loading regimens. Ragan ${ }^{23}$ reported transient upregulation of type II collagen within $4 \mathrm{~h}$ in 245 extracted bovine cartilage explants subjected to static mechanical compression but did not check the 246 chondrocyte survival rate. The current study, to our knowledge, is the first report on focal enhanced 247 staining of type II collagen in lesion cartilage that has undergone in vivo cyclic loading, despite the 248 complete death of the affected surface chondrocytes within 6 h. A previous study reported decreased 249 Safranin $\mathrm{O}$ and $\mathrm{Col} 2$ staining in an osteochondral defect model ${ }^{26}$, which was directly created on the AC 250 surface using a 1-mm biopsy punch. However, our model showed a diametrically opposite type-II251 collagen response to cyclic loading. Although one of the major collagenases, MMP-13, was overexpressed 252 immediately after loading injury (Figure 4), the morphological degradation of AC did not progress 253 extensively as in regular OA development. Further studies should focus on whether type II collagen 254 proliferation is beneficial or harmful to AC protection.

Lubricin localized on the cartilage surface is reportedly a protective and lubricating component of the $O$ linked glycoprotein ${ }^{27}$. We found drastic decrease in superficial lubricin staining of the damaged area immediately after cyclic loading compared with the non-loaded region (Figure 5). Decreased superficial 259 cartilage lubricin/proteoglycan 4 was confirmed in both in vivo ${ }^{28}$ and ex vivo ${ }^{29}$ experiments. Several 260 studies have reported an increased coefficient of friction within a few hours of cyclic loading ${ }^{30,31}$ and that 261 lubricin in the cartilage surface was denuded by loading, even in the joint where most chondrocytes 262 remained alive throughout the observation period ${ }^{31}$. Our study revealed similar results in a non-surgical 263 model during early observation after in vivo cyclic compression. On the other hand, after tracking different 264 time points for 8 weeks, we found that superficial cartilage staining with lubricin gradually recovered to 265 the normal level (Figure 8). The results after 4 weeks indicated a different trend of OA progression 
compared to that with joint instability-induced OA. Although lubricin expression on cartilage was found to be elevated in late-stage OA patients ${ }^{32}$, mainstream results from posttraumatic OA of human ${ }^{33}$ and animal joint instability models ${ }^{28,34-36}$ in a long-term observation demonstrated that joint lubricin concentration decreased after injury or surgery. Combining our results with the results of decreased $\operatorname{Prg} 4$ expression in unstable joints after forced movement, ${ }^{37,38}$ we hypothesized that instability-induced persistent motivation should play a more important role than magnitude of loading in determining irreversible lubricin loss. Several studies ${ }^{35,39,40}$ involving delivery of exogenous recombinant lubricin to medial meniscectomized rats found that the cartilage surface protein was protected from depletion in the experimental group. In the current study, we observed a self-healing process in the loading-damaged AC without any lubricin supplementation, indicating that endogenous lubricin is an important repair mechanism in the post-traumatic knee and providing a plausible explanation regarding the slower cartilage degradation progression in the non-invasive loading model compared to that in the joint instability surgery models. Interestingly, although superficial cell death was confirmed within $6 \mathrm{~h}$ after loading, the locations of cartilage lacunae were strongly stained with lubricin even at 8 weeks after injury. Previous studies found that chondrocytes encapsulated in agarose ${ }^{20,41}$ and cartilage explants ${ }^{42}$ expressed higher levels of prg4 when subjected to compressive strain and the intensified lacunae staining around cells was confirmed by immunofluorescence images ${ }^{20}$, similar to our results. Further studies should focus on potential links between chondrocyte-derived lubricin and the mechanism of superficial cartilage repair.

The current study has some limitations. First, it focused on investigating the medium-term changes over time from 3 days to 8 weeks after loading implementation. Since cell death, type II collagen biosynthesis, and superficial lubricin degradation occurred earlier than expected, further studies should more precisely design the unit of observation intervals in hours to reveal the full process of dynamic loading-induced 
289 cellular reactions. Second, in experimental sheep ${ }^{43,44}$ and horse $\mathrm{s}^{45,46}$ models, lubricin concentration in the 290 synovial fluid was upregulated transiently in the acute phase after injury, and synovial PRG4 (the gene 291 encoding lubricin) expression was positively correlated with TNF $\alpha$ and ADAMTS-5 ${ }^{46}$. Future studies 292 should evaluate not only cartilage, but also synovium using quantitative techniques to specify the details 293 of lubricin recovery. Moreover, we did not record the activities of rats after loading that may have 294 influenced the progression of lubricin self-healing, which may have contributed to the large variation in 295 the data (Figure 5D). Third, in the current study, we assessed only the cartilage lesion on the lateral femur 296 condyle, which is not the main part of cartilage loaded during walking ${ }^{47}$. Considering that cartilage 297 heterogeneity could affect the results of injury assessment, cartilage damage on the other contact surface should be examined in the future. Finally, we failed to compare the current model to any surgery-induced model. As described above, the surface lubricin is reportedly diminished in many injury-induced OA animals $^{28,34-36}$, whereas it is still unknown if joint instability independently affects the progression of post301 traumatic OA, especially in the lesion area. Further studies should combine invasive destabilization surgery with preexisting lesions caused by cyclic compression, which could reflect the spatiotemporal changes of cartilage in the non-contact area.

In conclusion, we found a specific, localized AC lesion in both the $20 \mathrm{~N}$ and $50 \mathrm{~N}$ groups that underwent 60 cycles of compression in rat knee joints. The local expression of type II collagen was increased after repeated loading, whereas lubricin in the cartilage surface was lost in response to cyclic compression. However, the distribution of superficial lubricin recovered 4 weeks after non-surgical injury (Figure 6). 308 These results indicate that dynamic loading exceeding $20 \mathrm{~N}$ damages the lateral femoral condyle AC in rats. Although the damage caused localized chondrocyte death and upregulated expression of degrading enzymes, endogenous repair in well-structured joints rebuilt the layer of protective proteins on the 311 superficial cartilage. 


\section{Acknowledgments}

This study was supported in part by a JSPS KAKENHI Grant, number JP18H03129 and JP18K19739.

\section{Author Contributions}

$\mathrm{XJ}$ : conception and design of the study, acquisition, analysis, and interpretation of data, drafting of the article, revision of the article, final approval of the article.

AI: conception and design of the study, interpretation of data, drafting of the article, revision of the article,

AN: conception and design of the study, interpretation of data, revision of the article, and final approval of the article.

$\mathrm{KN}$ : conception and design of the study, interpretation of data, revision of the article, final approval of the article.

HK: obtaining funding, conception and design of the study, interpretation of data, revision of the article, and final approval of the article.

TA: conception and design of the study, interpretation of data, revision of the article, and final approval

\section{References}

1. Kuyinu EL, Narayanan G, Nair LS, et al. Animal models of osteoarthritis: classification, update, and measurement of outcomes. J Orthop Surg Res 2016; 11: 19.

2. De Souza RL, Matsuura M, Eckstein F, et al. Non-invasive axial loading of mouse tibiae increases cortical bone formation and modifies trabecular organization: a new model to study cortical and cancellous compartments in a single loaded element. Bone 2005; 37: 810-818.

3. Holguin N, Brodt MD, Sanchez ME, et al. Adaptation of tibial structure and strength to axial compression depends on loading history in both C57BL/6 and BALB/c mice. Calcif Tissue Int 2013; 93: 211-221.

4. Lynch ME, Main RP, Xu Q, et al. Cancellous bone adaptation to tibial compression is not sex dependent in growing mice. J Appl Physiol (1985) 2010; 109: 685-691.

5. Poulet B, Hamilton RW, Shefelbine S, et al. Characterizing a novel and adjustable noninvasive murine joint loading model. Arthritis Rheum 2011; 63: 137-147.

6. Wu P, Holguin N, Silva MJ, et al. Early response of mouse joint tissue to noninvasive knee injury suggests treatment targets. Arthritis Rheumatol 2014; 66: 1256-1265.

7. Lockwood KA, Chu BT, Anderson MJ, et al. Comparison of loading rate-dependent injury modes in a murine model of post-traumatic osteoarthritis. J Orthop Res 2014; 32: 79-88. 
8. Ko FC, Dragomir C, Plumb DA, et al. In vivo cyclic compression causes cartilage degeneration and subchondral bone changes in mouse tibiae. Arthritis Rheum 2013; 65: 1569-1578.

9. Poulet B, de Souza R, Kent AV, et al. Intermittent applied mechanical loading induces subchondral bone thickening that may be intensified locally by contiguous articular cartilage lesions. Osteoarthritis Cartilage 2015; 23: 940-948.

10. Onur TS, Wu R, Chu S, et al. Joint instability and cartilage compression in a mouse model of posttraumatic osteoarthritis. J Orthop Res 2014; 32: 318-323.

11. Ko FC, Dragomir CL, Plumb DA, et al. Progressive cell-mediated changes in articular cartilage and bone in mice are initiated by a single session of controlled cyclic compressive loading. $\mathrm{J}$ Orthop Res 2016; 34: 1941-1949.

12. Adebayo OO, Ko FC, Wan PT, et al. Role of subchondral bone properties and changes in development of load-induced osteoarthritis in mice. Osteoarthritis Cartilage 2017; 25: 2108-2118.

13. McErlain DD, Appleton CT, Litchfield RB, et al. Study of subchondral bone adaptations in a rodent surgical model of OA using in vivo micro-computed tomography. Osteoarthritis Cartilage 2008; 16: 458-469.

14. Iijima H, Aoyama $\mathrm{T}$, Ito A, et al. Destabilization of the medial meniscus leads to subchondral bone defects and site-specific cartilage degeneration in an experimental rat model. Osteoarthritis Cartilage 2014; 22: 1036-1043.

15. Bomsta BD, Bridgewater LC, Seegmiller RE. Premature osteoarthritis in the Disproportionate micromelia (Dmm) mouse. Osteoarthritis Cartilage 2006; 14: 477-485.

16. Iijima H, Aoyama T, Ito A, et al. Effects of short-term gentle treadmill walking on subchondral bone in a rat model of instability-induced osteoarthritis. Osteoarthritis Cartilage 2015; 23: 15631574.

17. Hayami T, Pickarski M, Wesolowski GA, et al. The role of subchondral bone remodeling in osteoarthritis: reduction of cartilage degeneration and prevention of osteophyte formation by alendronate in the rat anterior cruciate ligament transection model. Arthritis Rheum 2004; 50: 1193-1206.

18. Iijima H, Aoyama T, Tajino J, et al. Subchondral plate porosity colocalizes with the point of mechanical load during ambulation in a rat knee model of post-traumatic osteoarthritis. Osteoarthritis Cartilage 2016; 24: 354-363.

19. Wong M, Carter DR. Articular cartilage functional histomorphology and mechanobiology: a research perspective. Bone 2003; 33: 1-13.

20. Jeon JE, Schrobback K, Hutmacher DW, et al. Dynamic compression improves biosynthesis of human zonal chondrocytes from osteoarthritis patients. Osteoarthritis Cartilage 2012; 20: 906-915.

21. Jorgensen AEM, Kjaer M, Heinemeier KM. The Effect of Aging and Mechanical Loading on the Metabolism of Articular Cartilage. J Rheumatol 2017; 44: 410-417.

22. Glasson SS, Askew R, Sheppard B, et al. Deletion of active ADAMTS5 prevents cartilage degradation in a murine model of osteoarthritis. Nature 2005; 434: 644-648.

23. Ragan PM, Badger AM, Cook M, et al. Down-regulation of chondrocyte aggrecan and type-II collagen gene expression correlates with increases in static compression magnitude and duration. J Orthop Res 1999; 17: 836-842.

24. Fitzgerald JB, Jin M, Dean D, et al. Mechanical compression of cartilage explants induces multiple time-dependent gene expression patterns and involves intracellular calcium and cyclic AMP. J Biol Chem 2004; 279: 19502-19511.

25. Anderson DE, Johnstone B. Dynamic Mechanical Compression of Chondrocytes for Tissue Engineering: A Critical Review. Front Bioeng Biotechnol 2017; 5: 76. 
26. Yamaguchi S, Aoyama T, Ito A, et al. The Effect of Exercise on the Early Stages of Mesenchymal Stromal Cell-Induced Cartilage Repair in a Rat Osteochondral Defect Model. PLoS One 2016; 11: e0151580.

27. Coles JM, Zhang L, Blum JJ, et al. Loss of cartilage structure, stiffness, and frictional properties in mice lacking PRG4. Arthritis Rheum 2010; 62: 1666-1674.

28. Elsaid KA, Machan JT, Waller K, et al. The impact of anterior cruciate ligament injury on lubricin metabolism and the effect of inhibiting tumor necrosis factor alpha on chondroprotection in an animal model. Arthritis Rheum 2009; 60: 2997-3006.

29. Jones AR, Chen S, Chai DH, et al. Modulation of lubricin biosynthesis and tissue surface properties following cartilage mechanical injury. Arthritis Rheum 2009; 60: 133-142.

30. McCann L, Ingham E, Jin Z, et al. Influence of the meniscus on friction and degradation of cartilage in the natural knee joint. Osteoarthritis Cartilage 2009; 17: 995-1000.

31. Drewniak EI, Jay GD, Fleming BC, et al. Cyclic loading increases friction and changes cartilage surface integrity in lubricin-mutant mouse knees. Arthritis Rheum 2012; 64: 465-473.

32. Neu CP, Reddi AH, Komvopoulos K, et al. Increased friction coefficient and superficial zone protein expression in patients with advanced osteoarthritis. Arthritis Rheum 2010; 62: 2680-2687.

33. Elsaid KA, Fleming BC, Oksendahl HL, et al. Decreased lubricin concentrations and markers of joint inflammation in the synovial fluid of patients with anterior cruciate ligament injury. Arthritis Rheum 2008; 58: 1707-1715.

34. Wei L, Fleming BC, Sun X, et al. Comparison of differential biomarkers of osteoarthritis with and without posttraumatic injury in the Hartley guinea pig model. J Orthop Res 2010; 28: 900-906.

35. Flannery CR, Zollner R, Corcoran C, et al. Prevention of cartilage degeneration in a rat model of osteoarthritis by intraarticular treatment with recombinant lubricin. Arthritis Rheum 2009; 60: 840-847.

36. Young AA, McLennan S, Smith MM, et al. Proteoglycan 4 downregulation in a sheep meniscectomy model of early osteoarthritis. Arthritis Res Ther 2006; 8: R41.

37. Elsaid KA, Zhang L, Waller K, et al. The impact of forced joint exercise on lubricin biosynthesis from articular cartilage following ACL transection and intra-articular lubricin's effect in exercised joints following ACL transection. Osteoarthritis Cartilage 2012; 20: 940-948.

38. Teeple E, Jay GD, Elsaid KA, et al. Animal models of osteoarthritis: challenges of model selection and analysis. Aaps j 2013; 15: 438-446.

39. Vugmeyster Y, Wang Q, Xu X, et al. Disposition of human recombinant lubricin in naive rats and in a rat model of post-traumatic arthritis after intra-articular or intravenous administration. Aaps $\mathrm{j}$ 2012; 14: 97-104.

40. Jay GD, Fleming BC, Watkins BA, et al. Prevention of cartilage degeneration and restoration of chondroprotection by lubricin tribosupplementation in the rat following anterior cruciate ligament transection. Arthritis Rheum 2010; 62: 2382-2391.

41. Nugent GE, Aneloski NM, Schmidt TA, et al. Dynamic shear stimulation of bovine cartilage biosynthesis of proteoglycan 4. Arthritis Rheum 2006; 54: 1888-1896.

42. Schatti OR, Markova M, Torzilli PA, et al. Mechanical Loading of Cartilage Explants with Compression and Sliding Motion Modulates Gene Expression of Lubricin and Catabolic Enzymes. Cartilage 2015; 6: 185-193.

43. Barton KI, Ludwig TE, Achari Y, et al. Characterization of proteoglycan 4 and hyaluronan composition and lubrication function of ovine synovial fluid following knee surgery. J Orthop Res 2013; 31: 1549-1554. 


\begin{abstract}
438 44. Atarod M, Ludwig TE, Frank CB, et al. Cartilage boundary lubrication of ovine synovial fluid following anterior cruciate ligament transection: a longitudinal study. Osteoarthritis Cartilage 2015; 23: 640-647.

441

442

443

444

445

446

447

448

449

45. Antonacci JM, Schmidt TA, Serventi LA, et al. Effects of equine joint injury on boundary lubrication of articular cartilage by synovial fluid: role of hyaluronan. Arthritis Rheum 2012; 64: 2917-2926.

46. Reesink HL, Watts AE, Mohammed HO, et al. Lubricin/proteoglycan 4 increases in both experimental and naturally occurring equine osteoarthritis. Osteoarthritis Cartilage 2017; 25: 128137.

47. Zevenbergen L, Smith CR, Van Rossom S, et al. 2018. Cartilage defect location and stiffness predispose the tibiofemoral joint to aberrant loading conditions during stance phase of gait. PloS one 13:e0205842.
\end{abstract}

450

451

452

453

454

455

456

457

458

459

460

461

462

463

464

465

466

467

468

469

470

471 


\section{Figure legends}

473

474

475

476

477

478

479

480

481

482

483

484

485

486

487

488

489

490

491

492

493

494

495

496

497

498

499

500

501

502

503

504

505

506

507

508

509

Figure 1. Schematic diagram of the non-surgical cyclic compression model. A. Right knee of anesthetized rat fixed on a customized apparatus with the patella embedded in a loading dent. The indexed knee angle was set at a deep flexion of $140^{\circ}$. B. A full cycle of the loading regimen contained a $0.5 \mathrm{~s}$ peak load and a $10 \mathrm{~s}$ rest interval with the loading cup approaching at a speed of $1 \mathrm{~mm} / \mathrm{s}$. The preload of $5 \mathrm{~N}$ and peak load of $20 \mathrm{~N}$ or $50 \mathrm{~N}$ were set for samples in corresponding groups. C. Flow chart of sample allocation.

Figure 2. Chondrocyte vitality in superficial cartilage after $20 \mathrm{~N}$ compressive loading detection by calcein AM/ethd-1 staining. Representative fluorescent images demonstrate spatiotemporal cell death from 1 to $12 \mathrm{~h}$ after loading. Green and red channels illustrate the distribution of live and dead chondrocytes, respectively. A well-defined focal region without green-stained cells was observed at 6 and 12 h. Preloaded normal rat limbs were used as controls. Scale bar: $100 \mu \mathrm{m}$.

Figure 3. Changes in type II collagen expression in articular cartilage substrates after cyclic loading. A. Representative immunostained sections for type II collagen in the lesion areas of lateral condyle cartilage. The border of normal and degenerative cartilage is represented by a dashed, light-gray line. B. Differences in type II collagen expression in control and loaded samples. C. Average intensity of staining on the degenerative cartilage matrix was calculated on 8-bit grayscale images using ImageJ software. D. Percentage variation of intensity in the lesion area relative to the intact area on the same section. Relative intensity was calculated in an inverted 8-bit grayscale image by dividing the intensity in the intact area. Significant effect or interaction between time points or load levels; ${ }^{*} \mathrm{p}<0.01$. No symbol: not significant. Scale bar: $100 \mu \mathrm{m}$.

Figure 4. The distribution of MMP- $13^{+}$chondrocytes in articular cartilage. A. Representative histological sections immunostained for MMP $-13^{+}$in the lesion area and the adjoining zone. Black arrow heads indicate positive cells in the superficial and intermediate zone of cartilage, red arrow heads indicate positive cells under the tidemark. B. Comparison of positive cell number in intact cartilage with all loaded samples' adjoining area. C, D. Results of semi-quantitative analyses of positive cells within the adjoining (C) and under the lesion area (D). Results were normalized by dividing by the cartilage surface length $(\mathrm{mm})$. Significant results $(\mathrm{p}<0.05)$ of ANOVA analysis $\$$ : main effect of duration was presented on the top of each chart. Marginal means of each observational point were compared when $\$$ was found. ${ }^{*} p<0.05$, $* * p<0.01$. Scale bar: $100 \mu \mathrm{m}$.

Figure 5. Localization of lubricin in articular cartilage subjected to dynamic loading. A. Representative Lubricin/Proteoglycan4 immunostained image in the lesion area of lateral condyle and intact area under the lateral meniscus. Staining was weakened in the superficial substrates, whereas it was enhanced in the cartilage lacunae. B. Differences between control $(n=6)$, early observation ( 3 days and 1 week; $n=24)$, and 
510 later observation (2, 4, and 8 weeks; $n=36)$ of loaded samples were compared using Kruskal-Wallis $\mathrm{H}$ 511 tests. C. Staining intensity in the superficial layer of cartilage substrates. The ROI of superficial cartilage 512 were depicted with ImageJ software using the brush selection tool with $50 \mu \mathrm{m}$ width (Supplementary 513 figure 3). D. Relative intensity normalized with intact region staining in percentage. Significant results $514(p<0.05)$ of a two-way ANOVA are presented on the top of each chart; $\$$ : main effect of duration, $\Psi$ : 515 interaction effects. Stratified one-way ANOVA on each load level with multiple comparison were applied 516 whenever $\Psi$ was found. ${ }^{*} p<0.05,{ }^{*} p<0.01$. No symbol: not significant. Scale bar: $100 \mu \mathrm{m}$.

518 Figure 6. The illustration summarizes the findings of the current study. Localized Col2 was upregulated 519 within 3 days after loading and stable during observation. Superficial lubricin decreased immediately after 520 damage yet recovered gradually to the normal level. Safranin O staining in lesion cartilage weakened 521 continuously after injury until week 4. Hematoxylin-stained nuclei in the damaged area dissolved 522 completely 2 weeks after cyclic compression. 


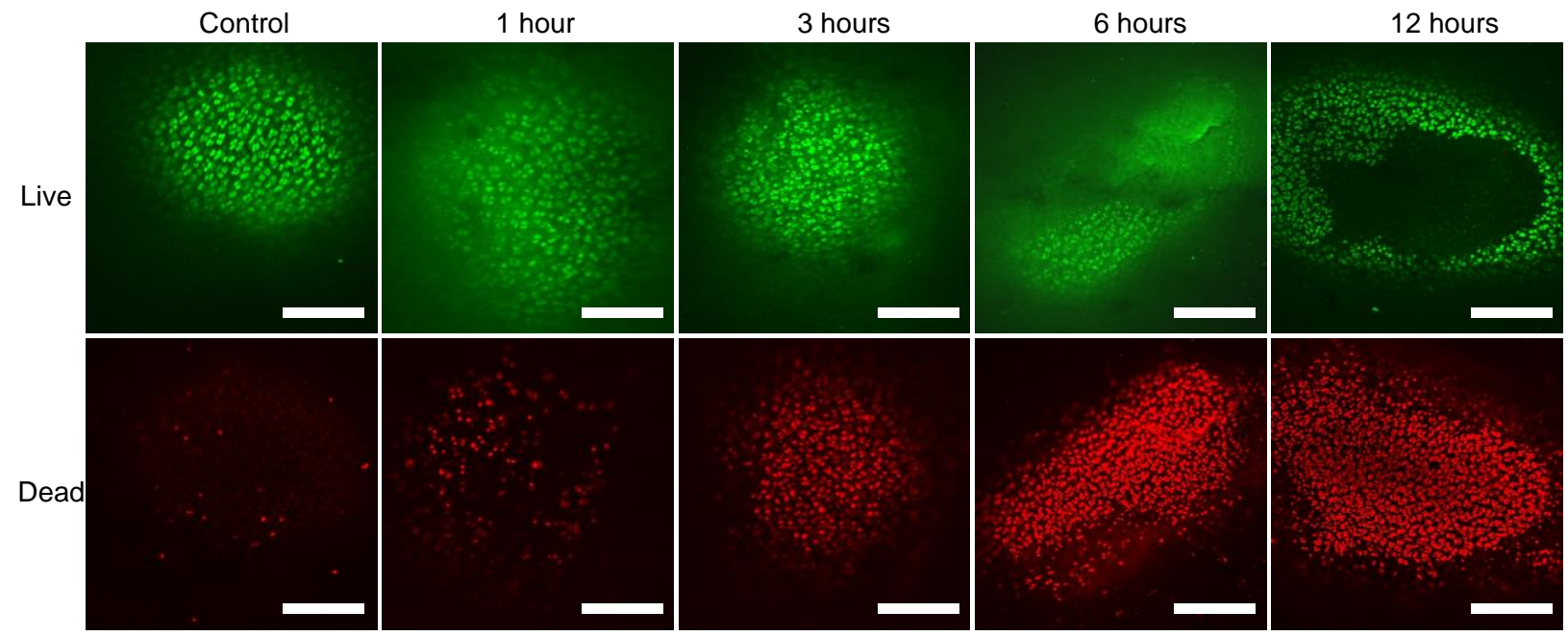




\section{的大学}

A $^{2}$

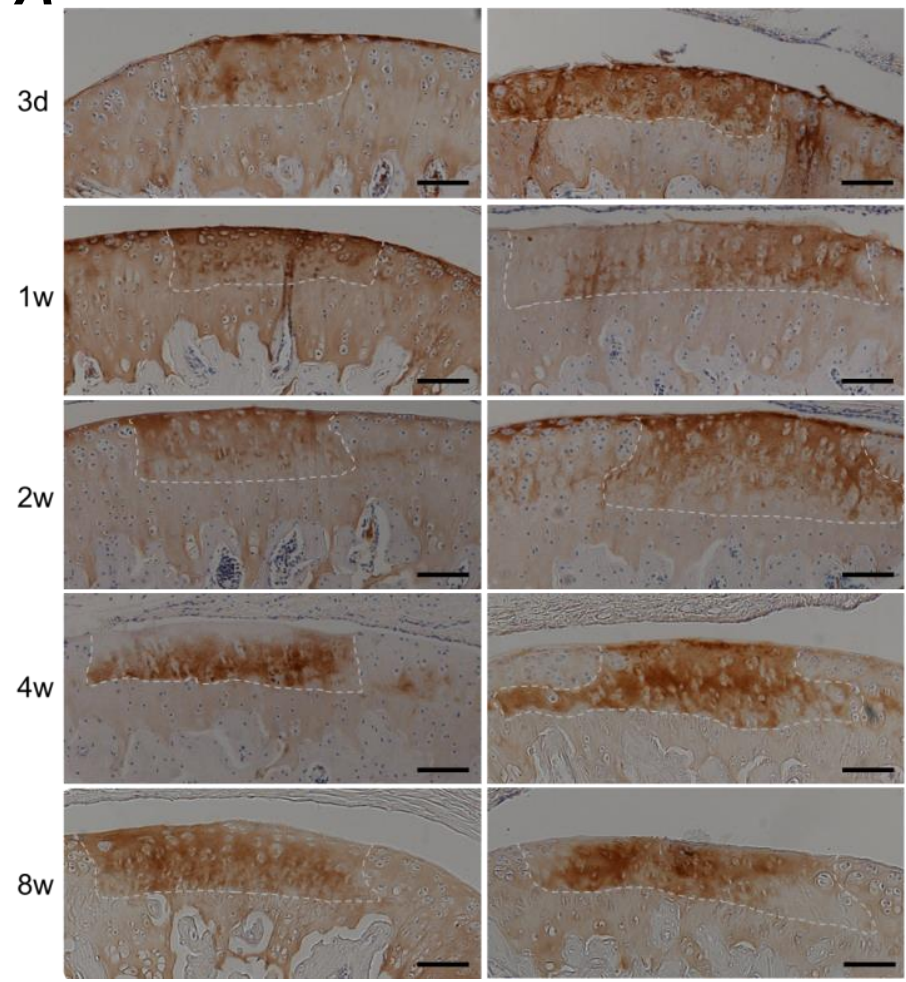

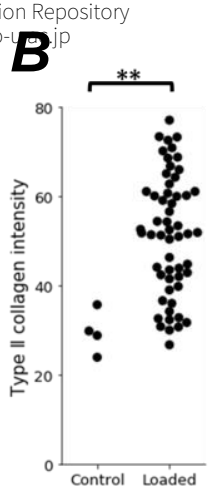

C

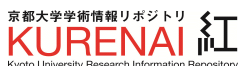

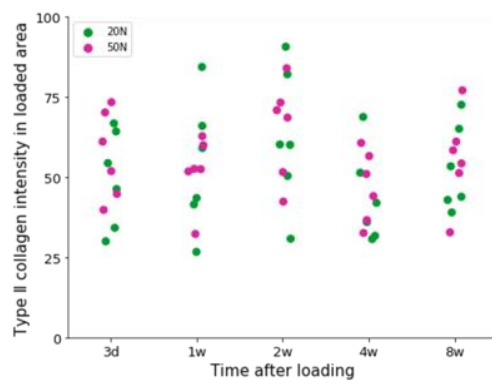

\section{D}

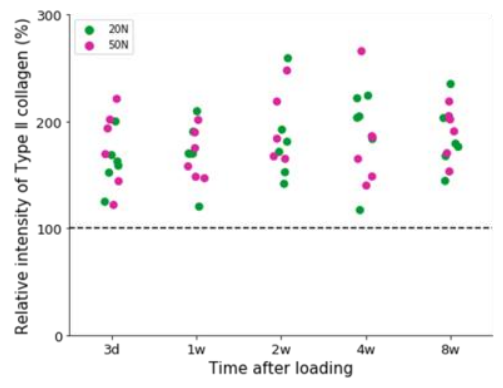


